\title{
Efficacy of glucocorticoids for the treatment of macrolide refractory mycoplasma pneumonia in children: meta- analysis of randomized controlled trials
}

Hwan Soo Kim ${ }^{1 \dagger}$, In Suk Sol ${ }^{2,3+}$, Donghe $\mathrm{Li}^{4}$, Miyoung Choi ${ }^{5}$, Yun Jung Choi ${ }^{6}$, Kyung Suk Lee ${ }^{7}$, Ju Hee Seo ${ }^{8}$, Yong Ju Lee ${ }^{9^{*}+}$, Hyeon-Jong Yang ${ }^{10^{* *}}$ (D) and Hyun Hee Kim ${ }^{1}$

\begin{abstract}
Background: Mycoplasma pneumoniae is one of the most common pathogens causing community acquired pneumonia in children. Although the rate of macrolide-refractory Mycoplasma pneumoniae (MRMP) has increased, systemic glucocorticoids as a treatment option has not been validated yet. The purpose of this study was to assess the efficacy of glucocorticoids add-on in the treatment of MRMP in children through systematic review and meta-analysis.

Methods: Data sources

A systematic literature search was conducted using ten electronic bibliographic databases including English, Korean, Chinese and Japanese languages, up to March 8, 2018.

Study selection

The study was conducted according to Preferred Reporting Items for Systematic Reviews and Meta-Analyses checklist and selected randomized control trials which compared the efficacy of glucocorticoids add-on to macrolide in the treatment of MRMP in children.

Data extraction

Two independent reviewers extracted: primary outcomes as hospital days, fever duration, and change in C-reactive protein (CRP) and main analysis was performed through meta-analysis with random effects model.

(Continued on next page)
\end{abstract}

\footnotetext{
* Correspondence: pedalllee@gmail.com; ilove902@hanmail.net

${ }^{\dagger}$ Hyeon-Jong Yang and Yong Ju Lee are co-corresponding authors.

${ }^{\dagger}$ Hwan Soo Kim and In Suk Sol are co-first authors.

${ }^{9}$ Department of Pediatrics, Hallym University Kangnam Sacred Heart Hospital,

Hallym University College of Medicine, 1 Singil-ro, Yeongdeungpo-gu, Seoul

07441, South Korea

${ }^{10}$ Pediatric Allergy and Respiratory Center, Department of Pediatrics, SCH

Biomedical Informatics Research Unit, Soonchunhyang University Seoul

Hospital, Soonchunhyang University College of Medicine, 59 Daesagwan-ro,

Yongsan-gu, Seoul 04401, South Korea

Full list of author information is available at the end of the article
}

(c) The Author(s). 2020 Open Access This article is distributed under the terms of the Creative Commons Attribution 4.0 International License (http://creativecommons.org/licenses/by/4.0/), which permits unrestricted use, distribution, and reproduction in any medium, provided you give appropriate credit to the original author(s) and the source, provide a link to the Creative Commons license, and indicate if changes were made. The Creative Commons Public Domain Dedication waiver (http://creativecommons.org/publicdomain/zero/1.0/) applies to the data made available in this article, unless otherwise stated. 


\begin{abstract}
(Continued from previous page)
Results: Twenty-four unique randomized controlled trials met the inclusion criteria. The mean length of hospital stay in glucocorticoids treatment group was significantly shorter than that in conventional macrolide-treatment group (Weighted mean difference $(W M D)=-4.03$ days). The mean length of fever duration was significantly shorter in the glucocorticoid treatment group in comparison with the conventional treatment group (WMD $=-3.32$ days). Level of CRP after treatment was significantly lower in the glucocorticoid treatment group than that in the conventional treatment group (WMD = -16.03). Sensitivity analysis and subgroup analysis showed no significant improvement in heterogeneity. As limitations of the study, most of the studies included were from a single country and we were unable to control for heterogeneity across interventions, lack of standardized measures, and different time points of assessments across studies.
\end{abstract}

Conclusions: Glucocorticoid add-on treatment for MRMP can significantly shorten the duration of fever and hospital stay and decrease the level of CRP. These results should be confirmed by adequately powered studies in the future.

Keywords: Pneumonia, Mycoplasma, Macrolides, Glucocorticoids

\section{Background}

Mycoplasma pneumoniae (M. pneumoniae) is one of the major pathogens causing community acquired pneumonia and bronchitis in children. Treatment of M. pneumoniaerelated respiratory infection is based on symptomatic treatment with antibiotics. Macrolides have been used as first line treatment. However, macrolide-refractory $M$. pneumoniae (MRMP) strains are increasing abruptly, particularly in East Asian countries including Korea, Japan, and China [1-3].

Most patients with MRMP do not show improvement of fever when they are treated with macrolides. Some may develop refractory or severe clinical course that requires additional treatment. Treatment for MRMP includes tetracyclines, fluoroquinolones, and systemic glucocorticoids [4].

Secondary antibiotics such as tetracyclines and fluoroquinolones are considered as effective alternatives in the treatment of MRMP. However, they are of limited use due to safety-concerns of teeth discoloration and musculoskeletal toxicity, particularly in children. Glucocorticoids can be also considered as alternative treatment options due to two reason. First, the pathogenesis of $M$. pneumoniae infection is associated with amplified host immune response and virulence of M. pneumoniae [5]. Second, adverse effects of glucocorticoids have been well established. Further risk is not likely to be added in the treatment of MRMP. However, previous studies on the effect of glucocorticoids have shown conflicting results [6-8].

The objective of this study was to assess the efficacy of glucocorticoids for treatment of MRMP in children through systematic review and meta-analysis.

\section{Methods}

Systematic Review and Meta-Analysis Protocols (PRISMA-P) 2015 were used for this systematic review and metaanalysis [9]. The Population-Intervention-ComparisonOutcome (PICO) question used for our search strategy was: "Does use of glucocorticoids help improve the outcome of MRMP in children?"

\section{Search strategy}

We performed a systematic search utilizing a protocol designed by two independent medical librarians (D.W.S. and M.L) specifically for this study with 10 electronic databases: PubMed, EMBASE, Cochrane Library, and Core journal (Korean, Japanese, and Chinese Journal) Full-text Database. The search encompassed articles published from January, 1990 to March 8, 2018. We used search terms listed in Additional file 1 to search PubMed, Cochrane, EMBASE, and database of core countries. We imposed no language or publication restrictions.

The first screening was executed by two independent reviewers (H.S.K. and I.S.S.) who evaluated the titles and abstracts obtained from the search. Records were managed using Endnote (version X8; Clarivate Analytics, Philadelphia, PA, USA). From this initial screening, articles that did not focus on glucocorticoid use in MRMP and review articles were immediately excluded. After initial exclusion process, full texts of the remaining articles were reviewed independently by two authors (H.S.K. and I.S.S.) to determine whether any articles met the predetermined eligibility criteria described in the next section. Disagreements between the two reviewers regarding the inclusion or exclusion of particular studies were settled by consultation with a third reviewer (Y.J.L.).

\section{Eligibility}

The following inclusion criteria were applied: (1) randomized control trial (RCT) which compare the efficacy of glucocorticoids add-on to macrolide alone in children with MRMP, (2) MRMP which was diagnosed with serology or polymerase chain reaction, and that refractories were defined clinically, (3) only included children $<18$ years of age, and (4) outcome measures with hospital days, fever duration, and level of C-reactive protein 
(CRP) change. Review articles, published abstracts without full-text publications, and case-study reports with 10 participants or less were excluded. Our search strategy included non-English articles in our initial search results. Non-English articles were then translated and included for evaluation.

\section{Study selection}

Two reviewers (H.S.K. and I.S.S.) independently screened titles and abstracts of the studies identified in our systematic search. Studies focusing on MRMP were included after review of abstracts. Full texts from included studies were reviewed to evaluate for eligibility. Reference lists of selected studies and previous reviews were also examined to determine any relevant publications overlooked by the electronic search. Disagreements between the two reviewers in the selection of particular studies were settled after discussion with a third reviewer (Y.J.L.).

\section{Risk of bias assessment}

Cochrane Collaboration Risk of Bias Tool was used by the two reviewers (H.S.K. and I.S.S.) who independently evaluated the risk of bias in included studies [10]. Risk of bias was determined as hi, low or unclear by evaluating random sequence generation, blinding of participants and personnel, incomplete outcome data, and selective reporting. Disagreements between the two reviewers regarding the risk of bias assessment of particular studies were settled after discussion with a third reviewer (Y.J.L.).

\section{Data extraction}

Two reviewers (H.S.K. and I.S.S.) used a structured form to extract data from each eligible study. Data extracted from each study could be characterized as characteristics of the sample, intervention details, and measurement of outcomes. Disagreements between the two reviewers regarding the data extraction of particular studies were settled by consultation with a third reviewer (Y.J.L.). Primary outcomes of the current study were hospital days, fever duration, and change in CRP.

\section{Statistical analysis}

The estimated mean effect of glucocorticoid add-on treatment on hospital days, fever duration, and change in C-reactive protein (CRP) and the associated 95\% confidence intervals (CIs) were extracted or calculated for the 24 studies included in the meta-analysis with Review Manager 5.3 (The Cochrane Collaboration, London, United Kingdom). Random-effects model was used for studies included in the analysis.

Heterogeneity was calculated using $\mathrm{I}^{2}$ statistic. The $\mathrm{I}^{2}$ statistic threshold should always be interpreted with care. A rough estimate of $25 \%$ denotes low heterogeneity while 50\% denotes moderate heterogeneity, and 75\% denotes high heterogeneity [11]. We conducted sensitivity analyses when heterogeneity was noted. This was performed by removing a study from the analysis to determine changes in $\mathrm{I}^{2}$ values and assess which studies play a significant role resulting in heterogeneity [11]. To assess the risk of publication bias, we used funnel plots for visual inspection, and Egger test and trim-and-fill method were performed for statistical identifying. All statistical

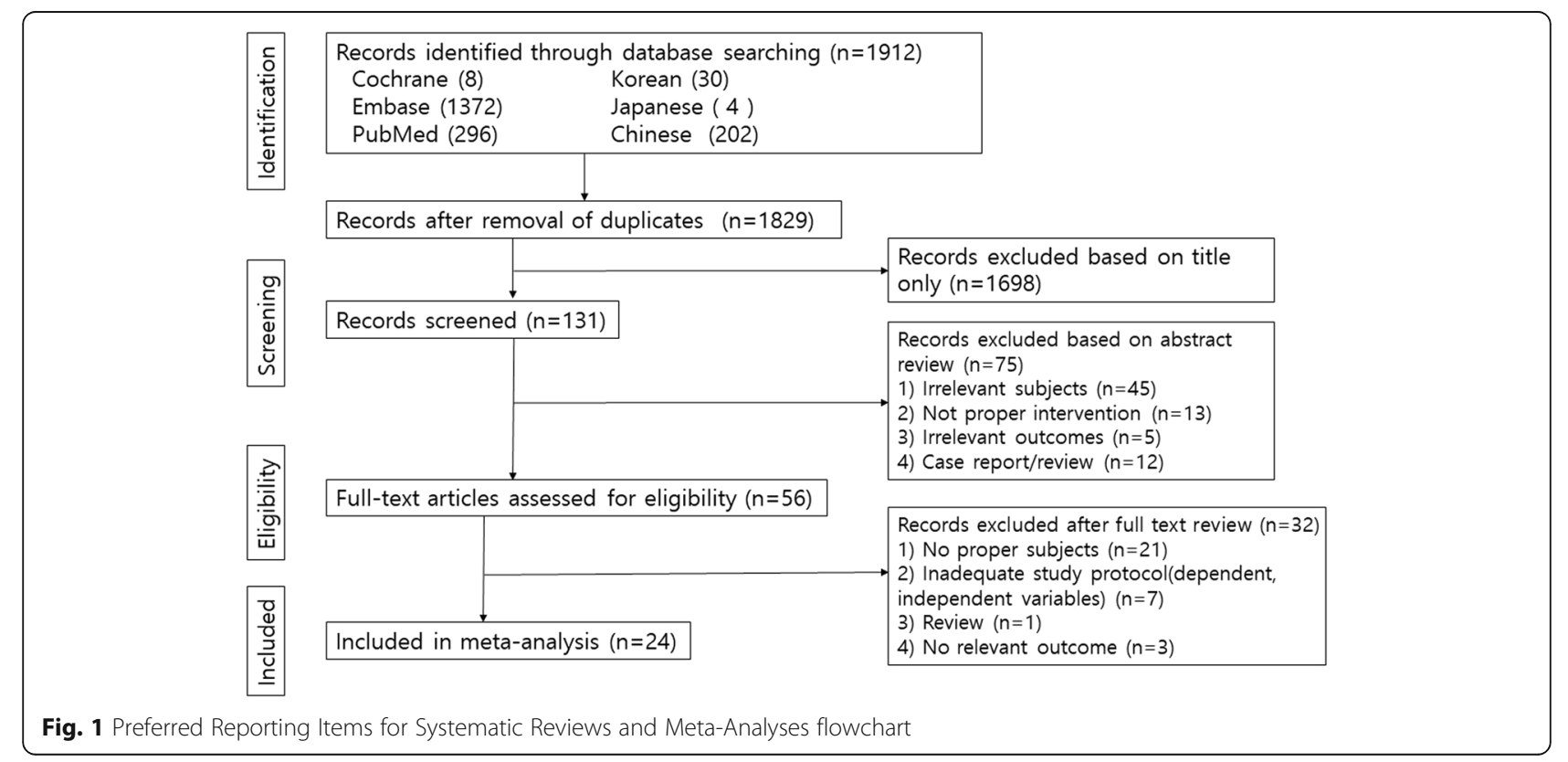




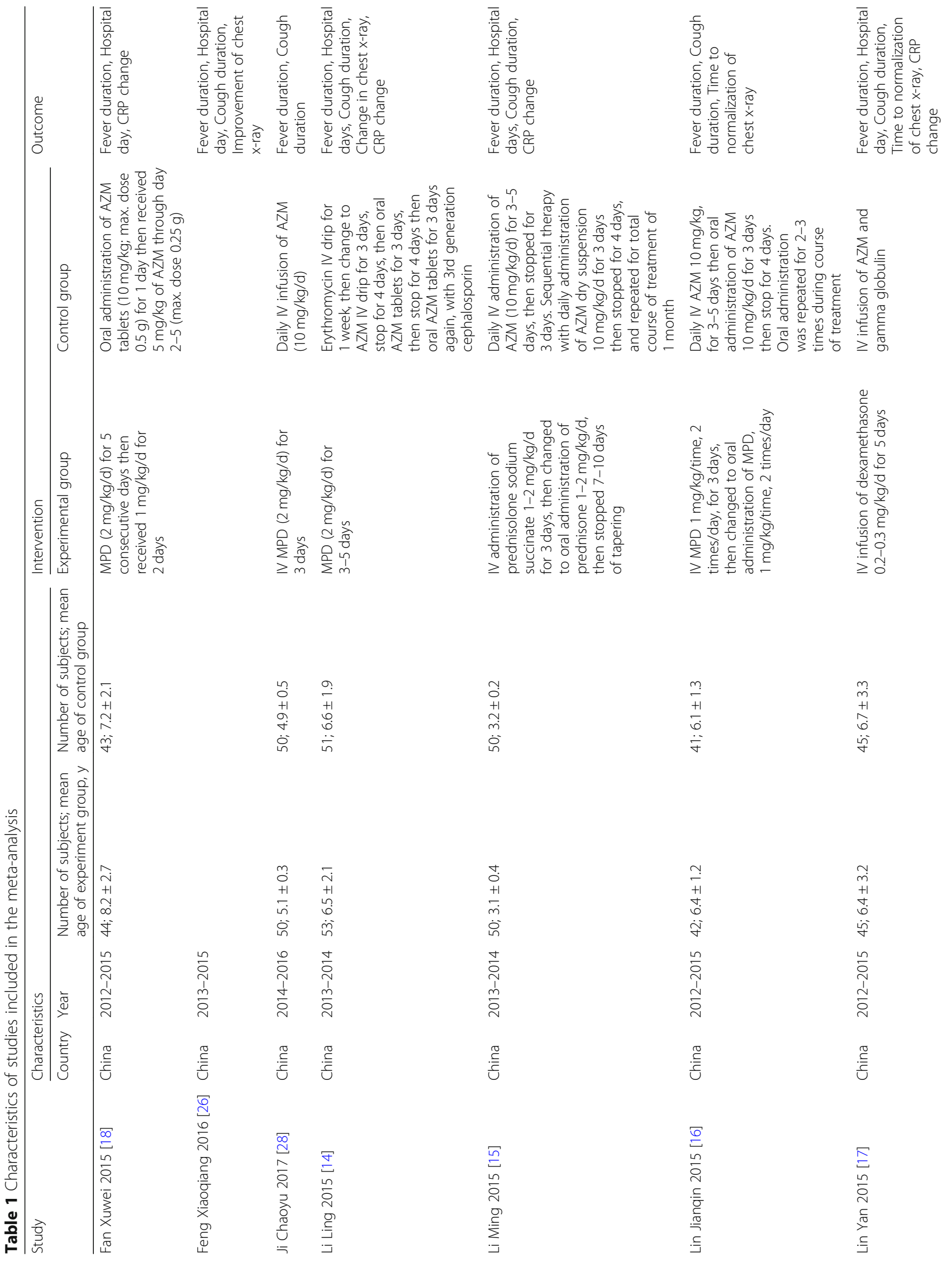


Kim et al. BMC Pulmonary Medicine ～～(2019) 19:251

Page 5 of 14

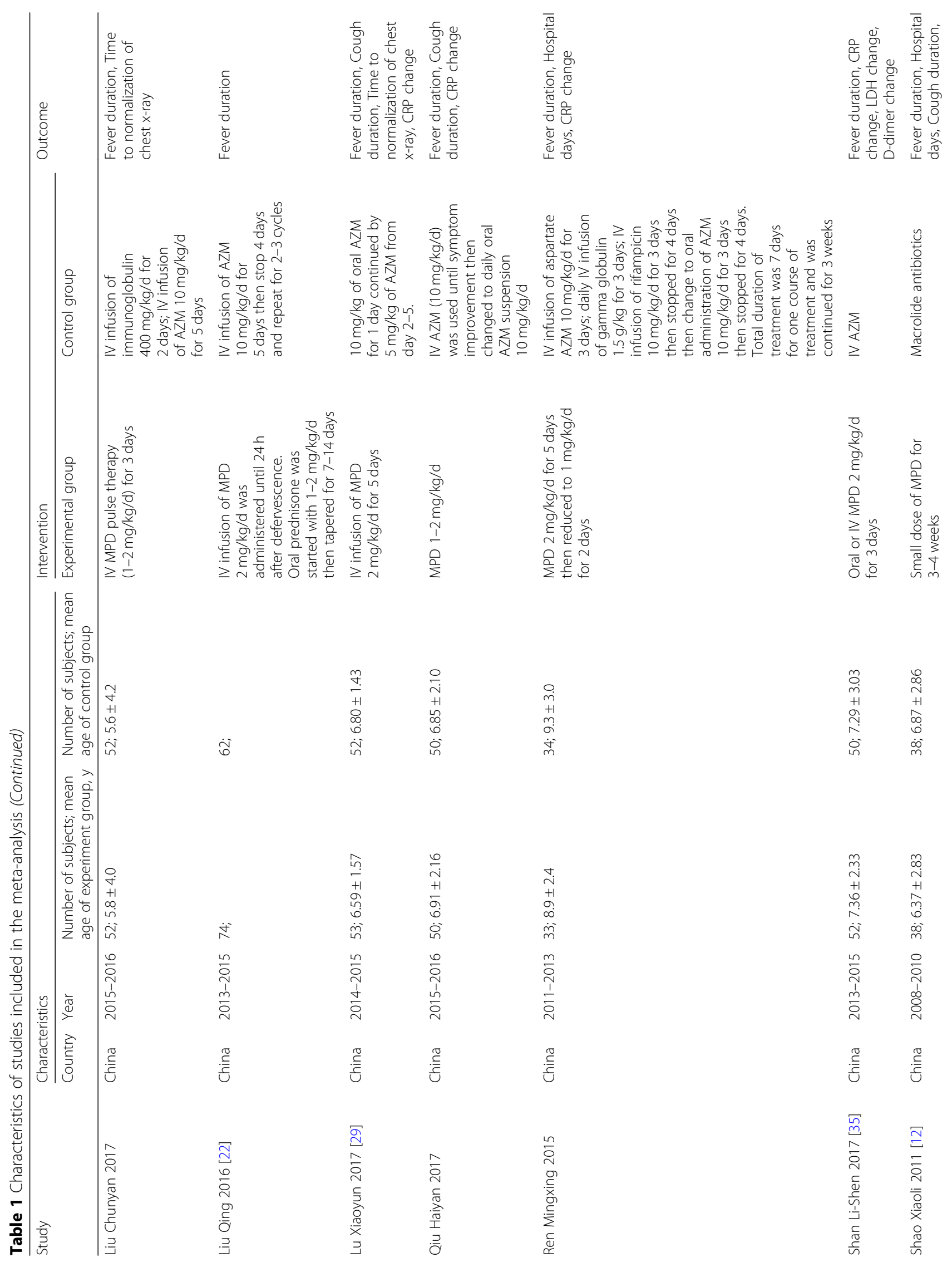




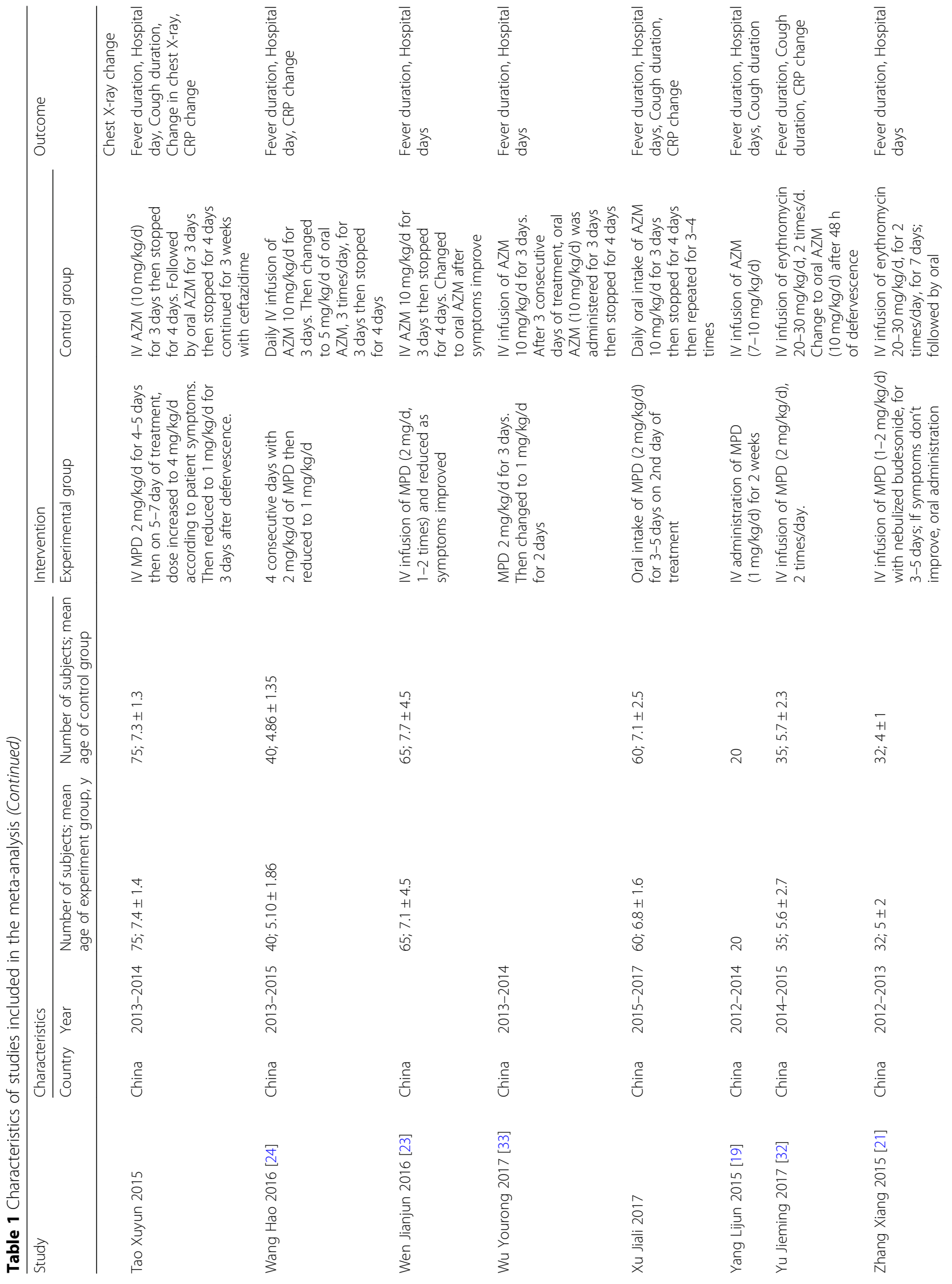




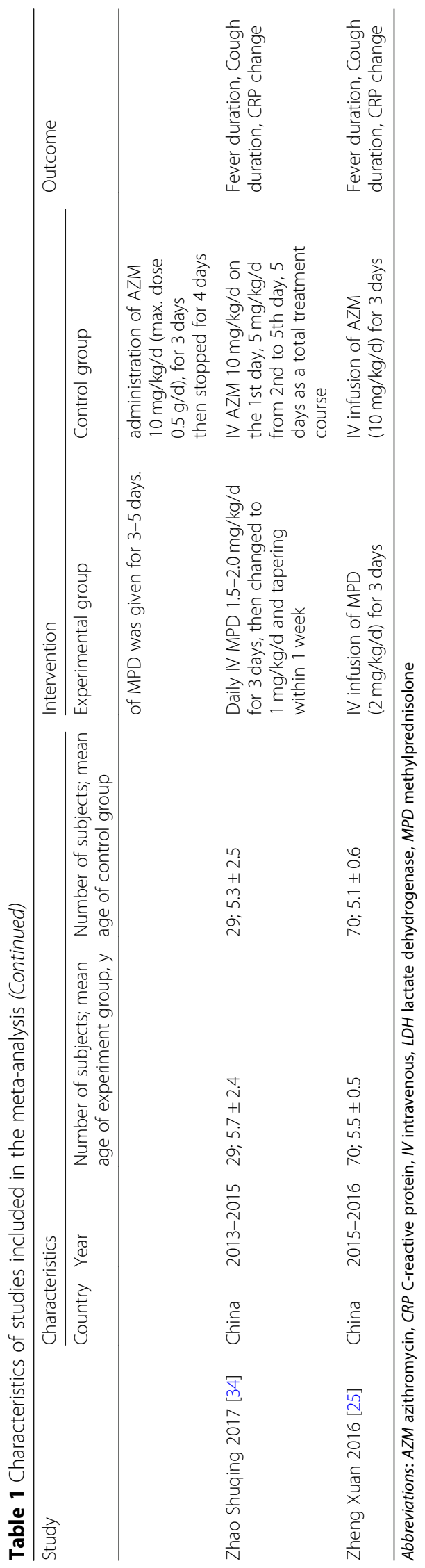


analyses were performed using $\mathrm{R}$ (version $3 \cdot 3 \cdot 3$ ) and Review Manager 5.3.

\section{Results}

\section{Systematic literature search results}

A total of 1829 citations were identified initially. Of these, 1773 studies were discarded after reviewing titles and abstracts, leaving 56 articles for full text review (Fig. 1). A total of 32 studies were excluded after full text review due to no proper subject, inadequate study protocol, review article, or no relevant outcome. A total of 24 studies were included in our systematic review and meta-analysis [12-35]. All studies were RCTs. (Additional file 1 for search strategies for database, Additional file 2 for PRISMA checklist).

\section{Sample characteristics}

Participants of studies enrolled in our meta-analysis was a total of 2365 patients. All these studies examined fever duration. Fifteen studies examined hospital days while 14 studies examined CRP level after treatment (Table 1).

\section{Fever duration}

The mean length of total duration of fever was significantly shorter in the glucocorticoid add-on group than that in the conventional treatment group (weighted mean difference, $\mathrm{WMD}=-3.32,95 \% \mathrm{CI}:-4.16--2.48, \mathrm{Z}=7.72$,
$P<0.00001)$. However, there was a high between-study heterogeneity of this effect $\left(\mathrm{I}^{2}=98 \%\right.$; Fig. 2$)$.

\section{Hospital days}

The mean length of hospital stays in the glucocorticoid add-on treatment group was significantly shorter than that in the conventional treatment group (WMD =4.03, 95\% CI: $-4.89--3.18, \mathrm{Z}=9.26, P<0.00001)$. However, there was a high between-study heterogeneity of this effect $\left(\mathrm{I}^{2}=90 \%\right.$; Fig. 3$)$.

\section{Reduction of CRP level after treatment}

The level of CRP after treatment was significantly lower in the glucocorticoid add-on treatment group than that in the conventional treatment group (WMD $=-16.03$, 95\%CI: $-22.56--9.50, Z=4.81, P<0.00001)$. However, there was a high between-study heterogeneity of this effect $\left(I^{2}=100 \%\right.$; Fig. 4$)$.

Sensitivity analyses was performed because of high level of heterogeneity. We removed a study from the analysis to determine which studies contributed most significantly to the heterogeneity by determining the changes in $\mathrm{I}^{2}$ values. We found that $\mathrm{I}^{2}$ values of fever duration, hospital days, and CRP level did not change.

\section{Subgroup analysis}

Use of glucocorticoids included the use of any type of glucocorticoids (e.g., methylprednisolone, dexamethasone, and

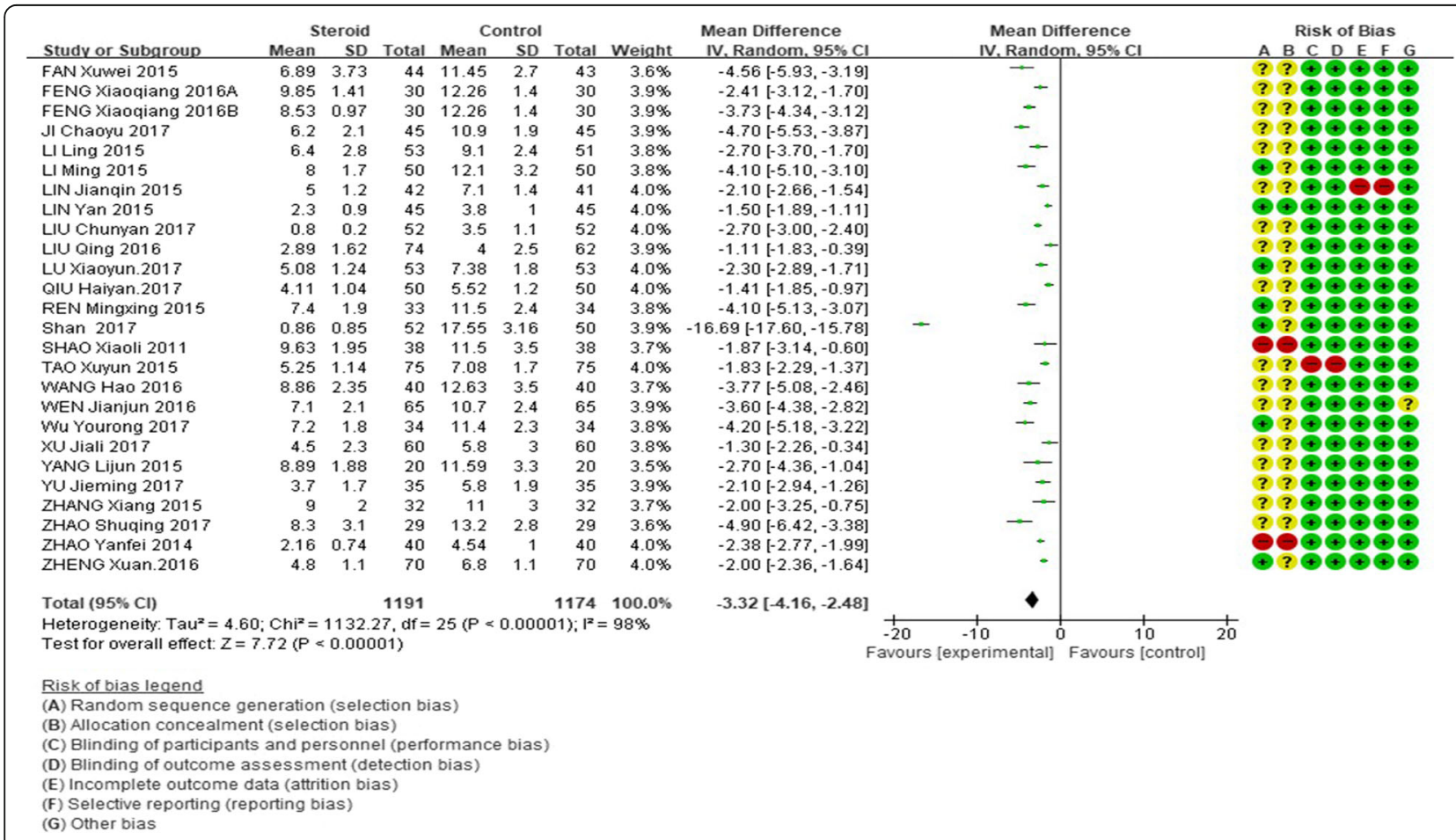

Fig. 2 Comparison of fever duration between glucocorticoid therapy and macrolide therapy 


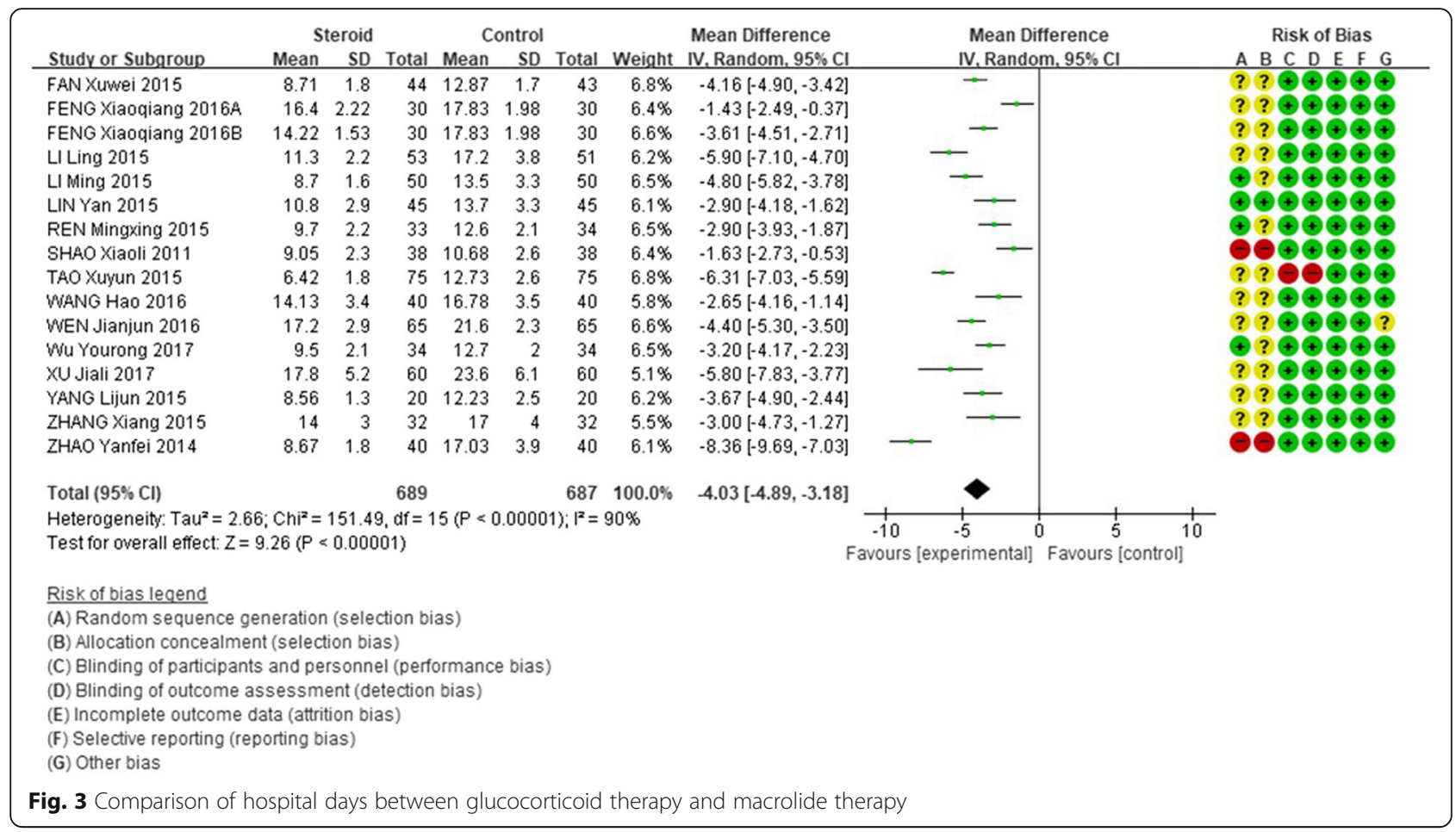

prednisolone). The use of different types of glucocorticoids was different across studies. This might have contributed to the heterogeneity in the overall use of glucocorticoids. Thus, we stratified the meta-analysis by subgroup analyses. In subgroup meta-analysis for use of methylprednisolone compared with the use of other glucocorticoids for the length of hospital stay, the use of other steroids did not show any significant subgroup differences (Figs. 5, 6 and 7).

\section{Publication bias}

All funnel plots were symmetric, indicating an absence of significant publication bias within these studies except

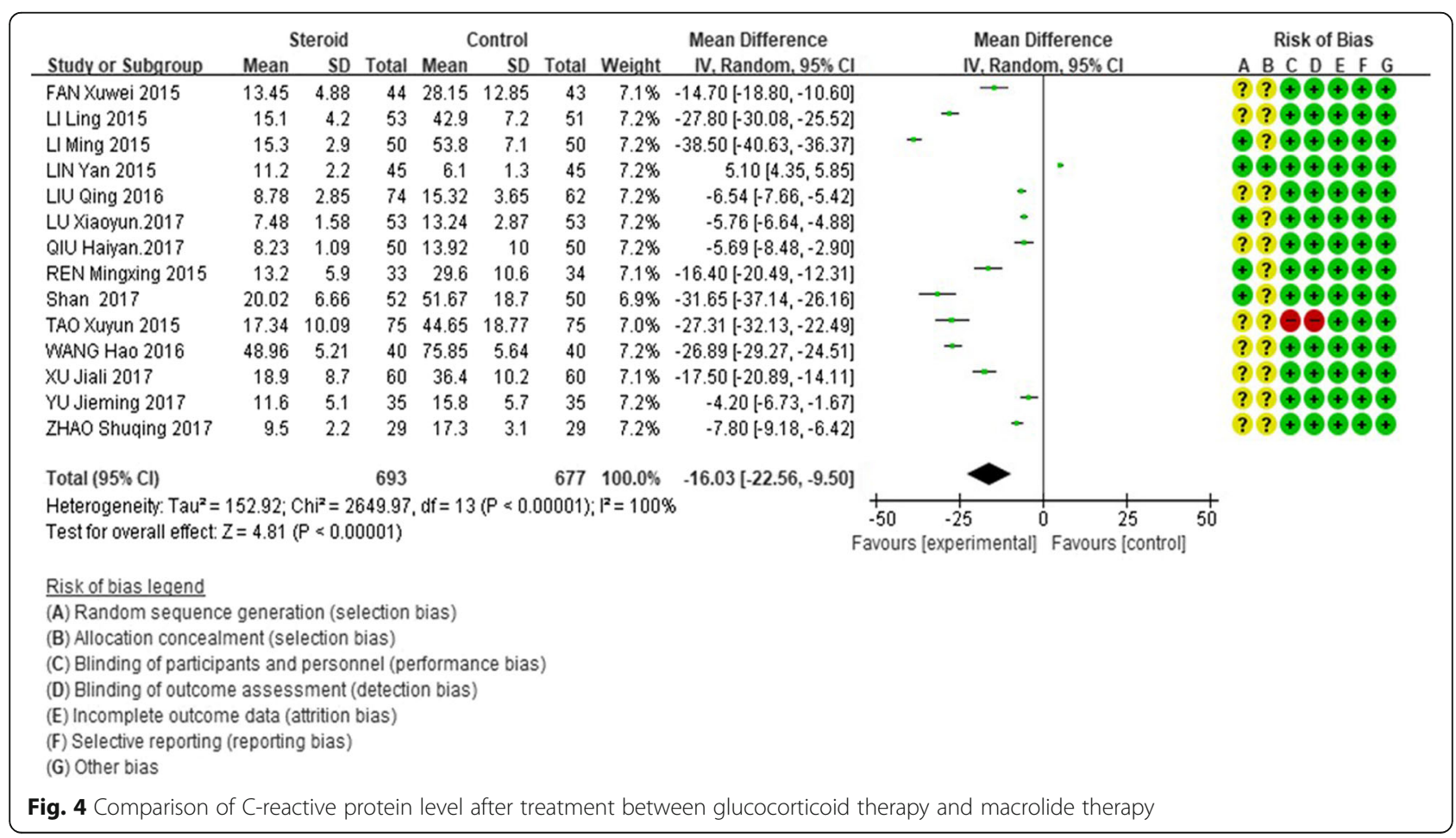




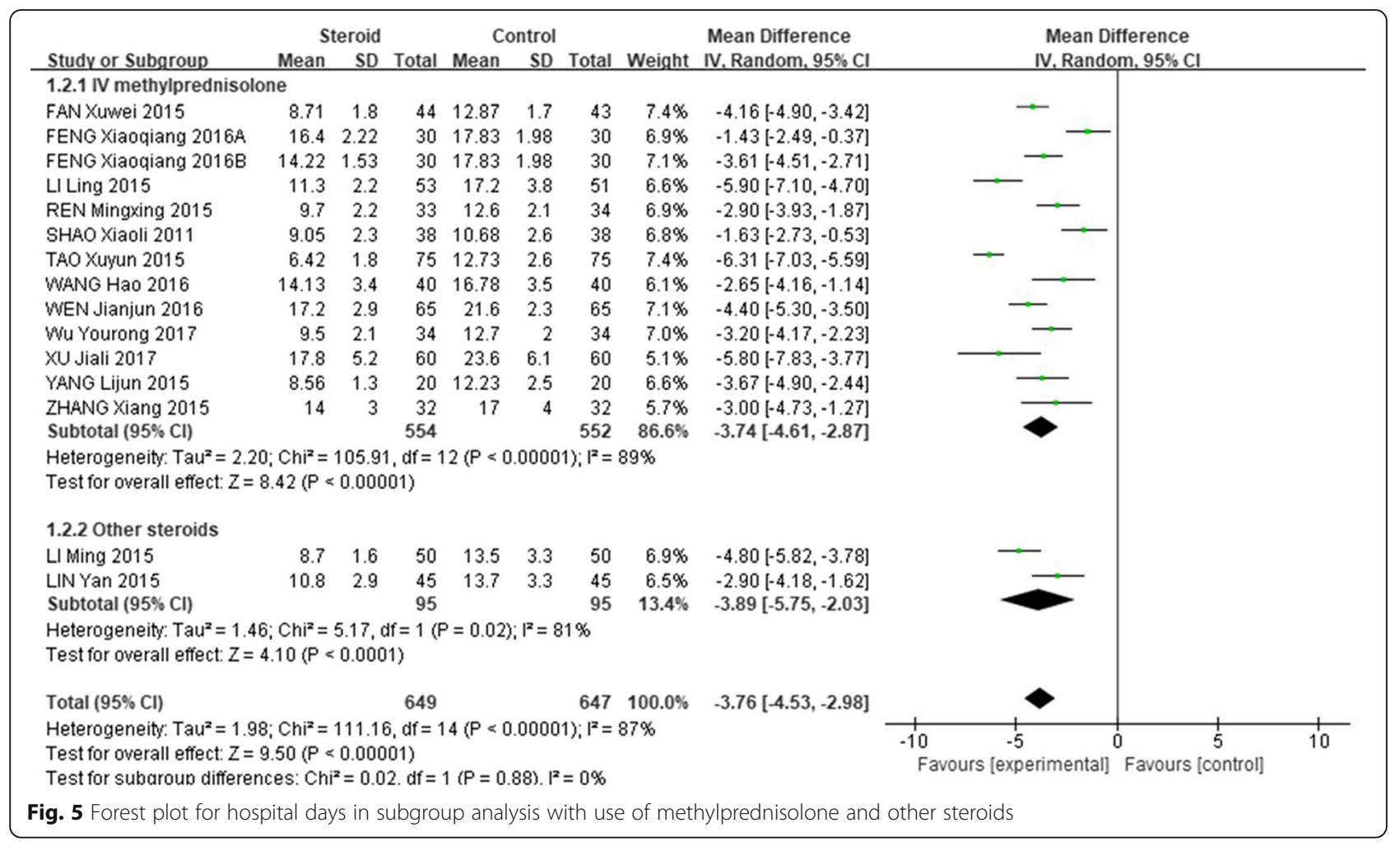

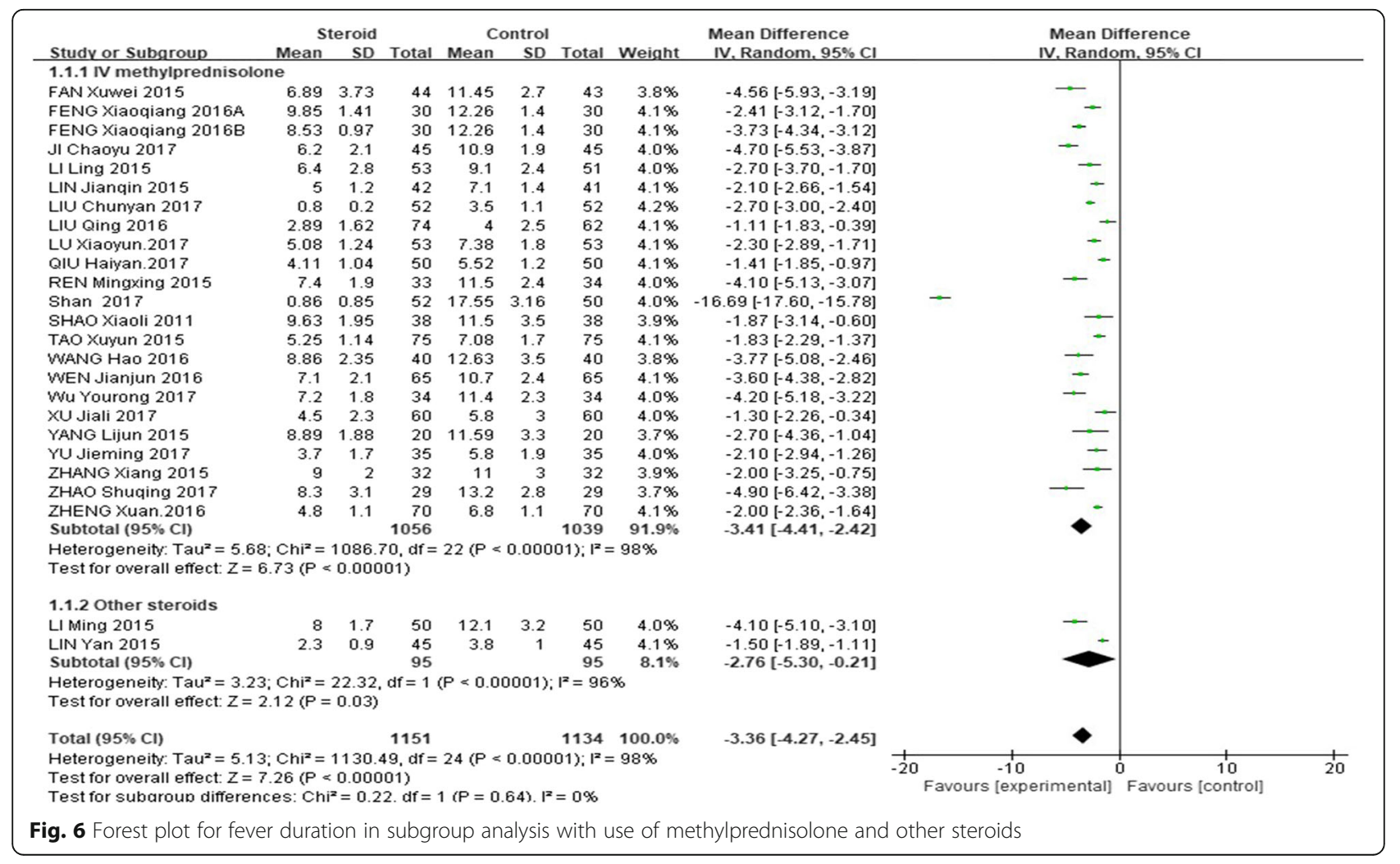




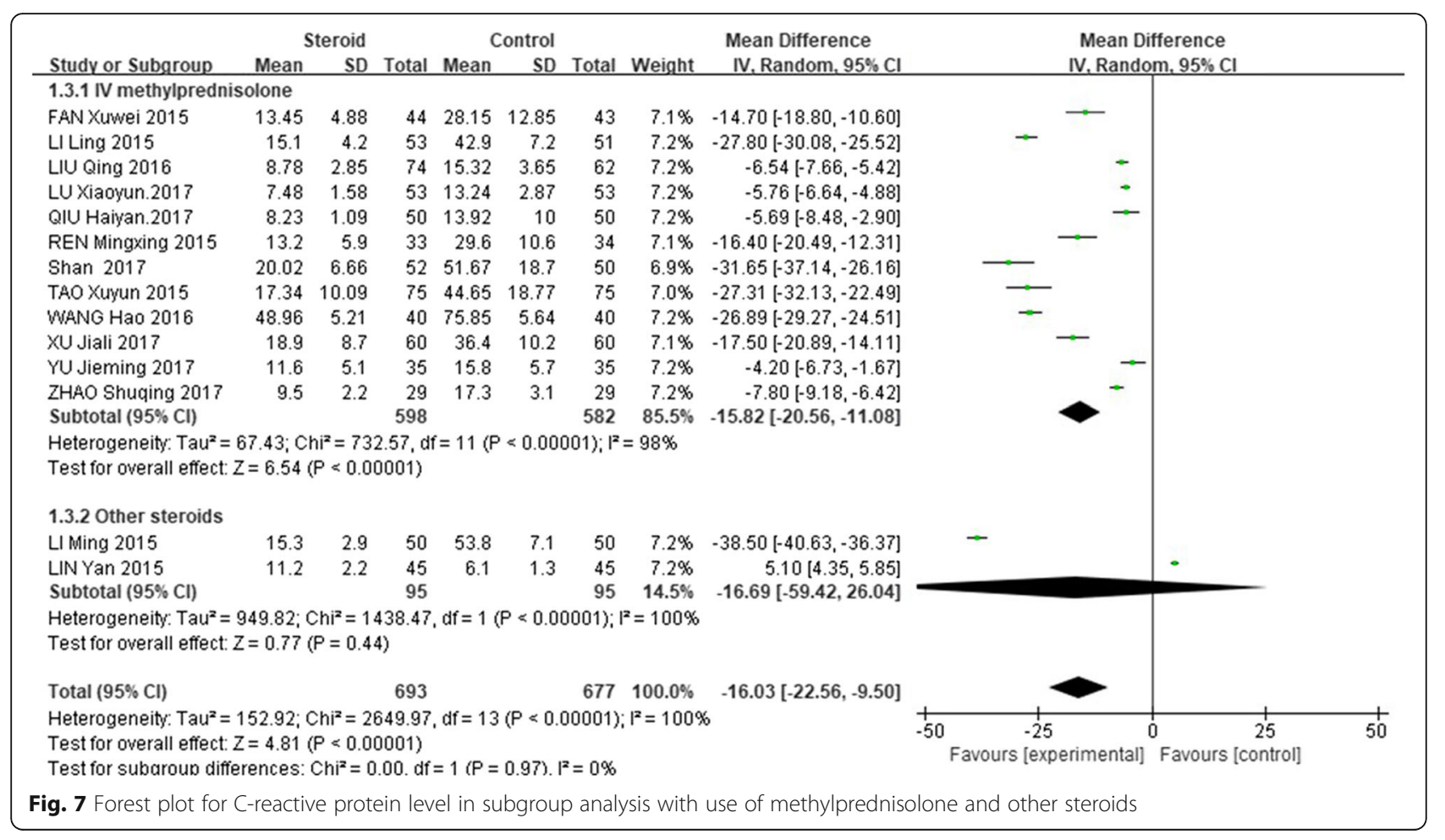

for CRP outcome. Egger test results were $-1.73(P=$ $0.09)$ for fever duration, $0.59(P=0.56)$ for hospital days, and - $3.19(P=0.008)$ for CRP. Trim-and-fill method for adjusting publication bias on CRP outcome was performed. The mean difference changed from $-3.27(P=$ $0.35)$ to $-16.03(P<0.001)$. These results indicated that there was substantial evidence of publication bias in CRP outcome (Fig. 8).

\section{Discussion}

This systematic review and meta-analysis identified and assessed RCTs on the use of glucocorticoids in children with MRMP. We specifically investigated effects of glucocorticoids on fever duration, length of hospital stay, and CRP level after treatment in comparison with conventional macrolide therapy. Results revealed positive effects of glucocorticoid treatment on all outcome measures.

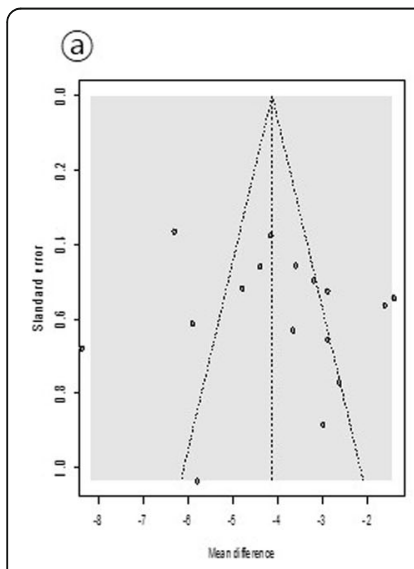

(b)

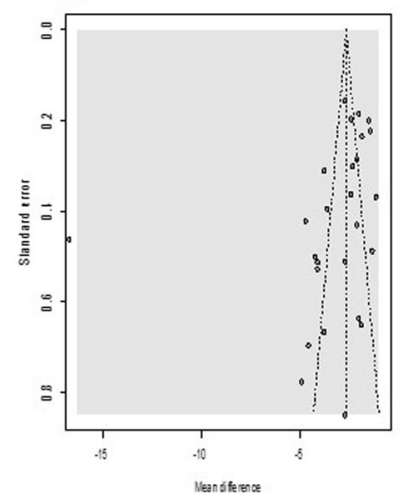

(c)

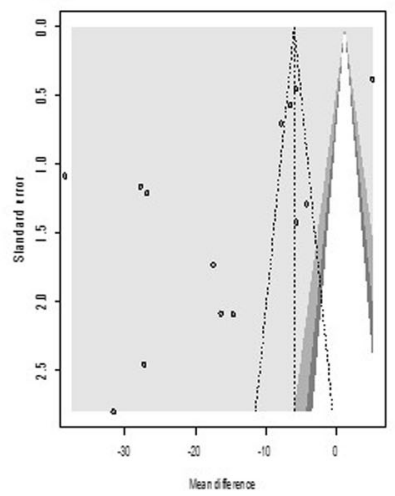

(d)

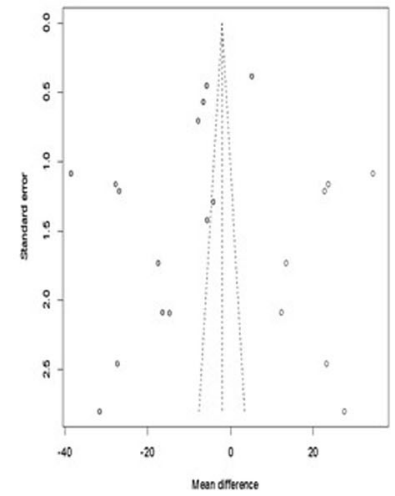

Fig. 8 Funnel plots showing WMDs and 95\% Cls for the efficacy of glucocorticoid therapy on the length of hospital stays (a), fever duration (b), and level of change of $\mathrm{C}$ reactive protein (c). Tests of asymmetry showed no significant deviation from the symmetry assumption $(\mathbf{a}, P=0.56 ; \mathbf{b}$, $P=0.06)$. However, significant asymmetry was found in the level of change of CRP $(\mathbf{c}, P=0.008)$. Trim-and-fill method for adjusting publication bias was used. Results showed substantial evidence of publication bias in CRP outcome (d) 
M. pneumoniae is a common pathogen causing community acquired pneumonia. The clinical course of $M$. pneumoniae infection is diverse, ranging from selflimiting to severe pneumonia with extra-pulmonary complications [36]. Macrolide is considered the first-line treatment for M. pneumoniae infection [37]. In adults, one study reported that prednisone treatment for 7 days in patients with community-acquired pneumonia admitted to hospital can shorten time to clinical stability without increase in complications [38]. However, another study reported that glucocorticoid use did not show any benefits in children [8]. Pulmonary injury associated with severe mycoplasma pneumonia could be caused by host immune response rather than by direct microbial damage [39, 40]. Overly active cell-mediated immunity and cytokine responses play a significant role in MRMP [41]. Severe M. pneumoniae infections and MRMP show similar laboratory findings with severe acute respiratory syndrome such as increased levels of non-specific markers of inflammation such as serum CRP, lactate dehydrogenase, and D-dimer [42]. Since MRMP can be considered as an immune-mediated disease, use of immune modulatory therapy could seem rational. For cases with MRMP infection, secondary treatment options are limited due to adverse effects of tetracycline or fluoroquinolones, especially in children [43]. The use of systemic steroids, in addition to antimicrobial therapy, to diminish host immune response in MRMP has been used in children and demonstrated satisfactory effects $[7,8,14]$. Glucocorticoids have shown to improve clinical features and reduce lung injury in children and adults $[5,44,45]$. The use of glucocorticoids in M. pneumoniae infection showed reduced lung histopathologic score by reducing cytokines and decreasing inflammatory response ultimately leading to lower mortality $[5,44,45]$. Therefore, glucocorticoids have been considered suitable as adjunctive therapy to macrolide therapy. Although previous studies have reported the efficacy of glucocorticoids in the treatment of severe MRMP, treatment protocols varied from oral prednisolone of $1 \mathrm{mg} / \mathrm{kg} /$ day to intravenous methylprednisolone $30 \mathrm{mg} / \mathrm{kg} /$ day for 3 days [7, 17]. These variable treatment protocols are due to the lack of guidelines for glucocorticoids treatment in severe MRMP to date $[7,46]$.

In our study, the mean length of total duration of fever, mean length of hospital stay, and level of CRP after treatment in the glucocorticoid treatment group were significantly shorter or lower than that those in the conventional treatment group. However, heterogeneity in all outcome measures was high and sensitivity analysis resulted in no significant difference. Subgroup analysis showed no significant decrease in heterogeneity. In spite of high level of heterogeneity, all studies reported favorable outcomes of hospital stays and fever duration after the use of glucocorticoids in MRMP. For CRP level, only one study reported unfavorable result after the use of glucocorticoids in MRMP. Therefore, we could consider that the use of glucocorticoids has significantly favorable effects on outcomes of MRMP.

The strength of this review is that it summarized the current evidence on the efficacy of glucocorticoids from RCT. Conducting RCTs offers the potential to produce the most definitive evidence to confirm or refute whether glucocorticoids can help improve outcomes of MRMP.

This review has some limitations. Firstly, almost all studies included in this review had at least one methodological flaw. Secondly, publication bias was significant for change in CRP level. It might be due to small sample sizes in most studies and the fact that most studies were conducted in a single country. As a result, the robustness of these outcomes should be explored with future studies. Thirdly, most of the studies included were from a single country. Lastly, we could not evaluate other outcomes such as improvement in chest $x$-ray infiltration or other symptoms such as cough because these data were not available in each study.

Despite these limitations, with our review and metaanalysis, this is the first study to date to synthesize the efficacy of glucocorticoids in MRMP.

\section{Conclusions}

In conclusion, even though some studies have reported the efficacy and effectiveness of systemic glucocorticoids in the treatment of MRMP [12-35], this is the first systematic review and meta-analysis to investigate the effectiveness of glucocorticoids in MRMP. We found that the use of glucocorticoids could shorten hospital days, shorten fever duration, and lower CRP levels after treatment.

However, these results should be interpreted cautiously, and future studies should also assess other outcomes to clarify the effect of glucocorticoids in MRMP.

\section{Supplementary information}

Supplementary information accompanies this paper at https://doi.org/10. 1186/s12890-019-0990-8

Additional file 1. Search strategies for database searching

Additional file 2. PRISMA checklist

\section{Abbreviations}

Cl: Confidence interval; CRP: C-reactive protein; M. pneumoniae: Mycoplasma pneumoniae; MRMP: Macrolide-refractory M. pneumoniae; PICO: PopulationIntervention-Comparison-Outcome; PRISMA-P: Systematic Review and MetaAnalysis Protocols; RCT: Randomized control trial; WMD: weighted mean difference 


\section{Acknowledgements}

We thank Misoon Lim and Dong Won Shin for providing assistance and support for the systematic search.

\section{Authors' contributions}

Study conception and design: HJY, YJL, HHK. Acquisition of data: HJY, YJL, DHL, MC, JHS. Analysis and interpretation of data: HSK, ISS, MC, HJY, YJL. Drafting of manuscript: HSK, DHL, YJC, KSL, JHS. Critical revision: HSK, ISS, KSL, HHK. All authors read and approved the final manuscript.

\section{Funding}

This research was supported by a grant from the Korea Health Technology R\&D Project through the Korea Health Industry Development Institute (KHIDI) funded by the Ministry of Health \& Welfare, Republic of Korea (grant number: HI16C2300). It was also supported by the Soonchunhyang University Research Fund. The funding source had no role in study design, data collection, data analysis, data interpretation, or writing of the report.

\section{Availability of data and materials}

The datasets used and/or analysed during the current study are available from the corresponding author on reasonable request.

\section{Ethics approval and consent to participate}

Not applicable.

\section{Consent for publication}

Not applicable.

\section{Competing interests}

The authors declare that they have no competing interests.

\section{Author details}

${ }^{1}$ Department of Pediatrics, College of Medicine, The Catholic University of Korea, Seoul, South Korea. ${ }^{2}$ Department of Pediatrics, Hallym University Chuncheon Sacred Heart Hospital, Chuncheon, Korea. ${ }^{3}$ Department of Pediatrics, Yonsei University College of Medicine, Seoul, Korea.

${ }^{4}$ Interdisciplinary Program of Bioinformatics, Seoul National University, Seoul, South Korea. ${ }^{5}$ Division of Health Technology Assessment Research, National Evidence-based Healthcare Collaborating Agency (NECA), Seoul, South Korea. ${ }^{6}$ Department of Pediatrics, Seoul National University Hospital, Seoul, Korea. ${ }^{7}$ Department of Pediatrics, Hanyang University Guri Hospital, Hanyang University College of Medicine, Guri, South Korea. ${ }^{8}$ Department of Pediatrics, Dankook University Hospital, Cheonan, South Korea. ${ }^{9}$ Department of Pediatrics, Hallym University Kangnam Sacred Heart Hospital, Hallym University College of Medicine, 1 Singil-ro, Yeongdeungpo-gu, Seoul 07441 South Korea. ${ }^{10}$ Pediatric Allergy and Respiratory Center, Department of Pediatrics, SCH Biomedical Informatics Research Unit, Soonchunhyang University Seoul Hospital, Soonchunhyang University College of Medicine, 59 Daesagwan-ro, Yongsan-gu, Seoul 04401, South Korea.

Received: 31 January 2019 Accepted: 13 November 2019 Published online: 18 December 2019

\section{References}

1. Lee $\mathrm{E}$, Cho HJ, Hong SJ, Lee J, Sung H, Yu J. Prevalence and clinical manifestations of macrolide resistant mycoplasma pneumoniae pneumonia in Korean children. Korean J Pediatr. 2017;60:151-7.

2. Meyer Sauteur PM, Unger WW, Nadal D, Berger C, Vink C, van Rossum AM Infection with and carriage of mycoplasma pneumoniae in children. Front Microbiol. 2016;7:329.

3. Yamazaki T, Kenri T. Epidemiology of mycoplasma pneumoniae infections in Japan and therapeutic strategies for macrolide-resistant M pneumoniae. Front Microbiol. 2016;7:693.

4. Yang HJ, Song DJ, Shim JY. Mechanism of resistance acquisition and treatment of macrolide-resistant mycoplasma pneumoniae pneumonia in children. Korean J Pediatr. 2017;60:167-74

5. Youn YS, Lee KY. Mycoplasma pneumoniae pneumonia in children. Korean Pediatr. 2012;55:42-7.

6. Meijvis SC, Hardeman $H$, Remmelts $H H$, Heijligenberg R, Rijkers GT, van Velzen-Blad $\mathrm{H}$, et al. Dexamethasone and length of hospital stay in patients with community-acquired pneumonia: a randomised, double-blind, placebo-controlled trial. Lancet. 2011:377:2023-30.

7. You SY, Jwa HJ, Yang EA, Kil HR, Lee JH. Effects of methylprednisolone pulse therapy on refractory mycoplasma pneumoniae pneumonia in children. Allergy Asthma Immunol Res. 2014;6:22-6.

8. Okubo Y, Michihata N, Morisaki N, Uda K, Miyairi I, Ogawa Y, et al. Recent trends in practice patterns and impact of corticosteroid use on pediatric mycoplasma pneumoniae-related respiratory infections. Respir Investig. 2018:56:158-65

9. Moher D, Liberati A, Tetzlaff J, Altman DG. Preferred reporting items for systematic reviews and meta-analyses: the PRISMA statement. PLoS Med. 2009;6:e1000097.

10. Higgins JP, Altman DG, Gotzsche PC, Juni $P$, Moher D, Oxman AD, et al. The Cochrane Collaboration's tool for assessing risk of bias in randomised trials. BMJ. 2011;343:d5928.

11. Higgins JP, Thompson SG, Deeks JJ, Altman DG. Measuring inconsistency in meta-analyses. BMJ. 2003:327:557-60.

12. Shao X-L, Liu S-M, Lou W-Y, Zhu G-Z. Methylprednisolone in adjunctive treatment of refractory mycoplasma pneumonia. Chin Gen Pract. 2011;14: $186-7$.

13. Xu-yun T, Hong-yu Q. Clinical effect and safety of small-dose methylprednisolone triple therapy on refractory lobar mycoplasma pneumoniae pneumonia in children. Pract J Card Cerebral Pneumal Vasc Dis. 2015;23:74-6.

14. Ling $L$. The effect of low-dose methylprednisolone in the treatment of children with refractory mycoplasma pneumoniae pneumonia. J Pediatr Pharm. 2015;21:18-21.

15. Ming $\mathrm{L}$, Ye-qing $\mathrm{H}$. Small doses of methyl prednisolone sodium succinate and prednisone combined treatment the curative effect of children refractory mycoplasma pneumoniae pneumonia. Medical Innovation of China. 2015;12:10-3.

16. Jianqin L. Evaluation clinical curative effect of methylprednisolone in treatment of 42 case children with refractory mycoplasma pneumonia. China Contin Med Educ. 2015;7:148-9.

17. Yan L, Wei L. Effect of low-dose glucocorticoid in the treatment of children with refractory mycoplasma pneumonia. China Mod Med. 2015;22:82-4.

18. Xuwei F. Methylprednisolone combined with azithromycin in treatment of children with refractory mycoplasma pneumonia (RMPP) efficacy and safety. Prog Biomed Eng. 2015;36:246-8.

19. Lijun Y. Clinical curative effect analysis of methyl prednisolone auxiliary treatment of refractory mycoplasma pneumonia. China Contin Med Educ. 2015;7:142-3.

20. Ming-xing R, Guochang X, Linna S, Huan X, Yuejuan S, Li C. Efficacy and safety of methylprednisolone combined with azithromycin on refractory mycoplasma pneumonia in children. Chin Gen Pract. 2015;18: 588-91.

21. Zhang $X$. Clinical study of glucocorticoid in the treatment of refractory mycoplasma pneumoniae pneumonia. J Clin Pulm Med. 2015;20:1472-5.

22. Qing L, Hu L, Ling Z, Yajun H. Effect of therapy with glucocorticoids in children with refractory mycoplasma pneumoniae pneumonia. J Pediatr Pharm. 2016;22:25-7.

23. Jianjun W. Study on the effect of azithromycin and methylprednisolone in the treatment of children with refractory mycoplasma pneumonia. Chin Community Dr. 2016:32:31-3.

24. Hao W, Jingrong $Y$, Jinglin $O$. Clinical effect of methylprednisolone combined with azithromycin in the treatment of children with RMPP. Med Sci J Cent South China. 2016;44:442-6.

25. Xuan Z, Juanjuan Z, Huichao J, Yang Z. Observation on efficacy of methylprednisolone in treatment of children with refractory mycoplasma pneumoniae. Eval Anal Drug-Use Hosp China. 2016;16:1351-3.

26. Xiaogiang F, Chunyan G, Xiaohong H, Shiping L. Clinical analysis of the effects of methylprednisolone on the treatment of refractory mycoplasma pneumoniae pneumonia in children. Transl Med J. 2016:5:92-5.

27. Hai-yan Q. To observe the curative effect of small dose of glucocorticoid combined with Pulmicort inhalation in the treatment of refractory mycoplasma pneumonia. Contemp Med. 2017;23:37-9.

28. Chaoyu J, Rongfang M. Hormone refractory mycoplasma pneumonia lavage cytokines. Shaanxi Med J. 2017;46:22-4.

29. Xiaoyun L, Jinping F, Qiaoziang X. Intravenous methylprednisolone combined with azithromycin in treatment of children with refractory mycoplasma pneumonia and influence on relevant laboratory parameters. J Pediatr Pharm. 2017;23:14-6. 
30. Chun-yan L, Ling-zhi C. Clinical effect of methylprednisolone pluse therapy in treatment of refractory mycoplasma pneumoniae in children. Syst Med. 2017;2:70-87.

31. Jia-li X, Ying W, Peng C. Clinical effect of methylprednisolone combined with azithromycin sequential therapy in treating children with mycoplasma pneumonia and the impact on serum levels CRP and STREM-1. Pract I Card Cereb Pneumal Vasc Dis. 2017;25:155-7.

32. Yu J, Wang $X$, Wu C. Clinical analysis of methylprednisolone in the treatment of children refractory mycoplasma pneumoniae pneumonia. China Pract Med. 2017;12:7-9.

33. Wu X. Analysis of the effect of combined use of methylprednisolone and azithromycin on refractory mycoplasma pneumonia in children. Contemp Med Symp. 2017;15:133-4.

34. Shuqing Z, Yanrong Z. Effect of small doses of methylprednisolone treatment of children with refractory mycoplasma. Med Recapitulate. 2017; 23:798-801.

35. Shan LS, Liu X, Kang XY, Wang F, Han XH, Shang YX. Effects of methylprednisolone or immunoglobulin when added to standard treatment with intravenous azithromycin for refractory mycoplasma pneumoniae pneumonia in children. World J Pediatr. 2017;13:321-7.

36. Defilippi A, Silvestri M, Tacchella A, Giacchino R, Melioli G, Di Marco E, et al. Epidemiology and clinical features of mycoplasma pneumoniae infection in children. Respir Med. 2008;102:1762-8.

37. Mandell LA, Wunderink RG, Anzueto A, Bartlett JG, Campbell GD, Dean NC, et al. Infectious Diseases Society of America/American Thoracic Society consensus quidelines on the management of community-acquired pneumonia in adults. Clin Infect Dis. 2007;44(Suppl 2):S27-72.

38. Blum CA, Nigro N, Briel M, Schuetz P, Ullmer E, Suter-Widmer I, et al. Adjunct prednisone therapy for patients with community-acquired pneumonia: a multicentre, double-blind, randomised, placebo-controlled trial. Lancet. 2015;385:1511-8.

39. Waites KB, Talkington DF. Mycoplasma pneumoniae and its role as a human pathogen. Clin Microbiol Rev. 2004;17:697-728.

40. Tanaka H, Narita M, Teramoto S, Saikai T, Oashi K, Igarashi T, et al. Role of interleukin-18 and T-helper type 1 cytokines in the development of mycoplasma pneumoniae pneumonia in adults. Chest. 2002;121:1493-7.

41. Shimizu T, Kida Y, Kuwano K. Cytoadherence-dependent induction of inflammatory responses by mycoplasma pneumoniae. Immunology. 2011; 133:51-61.

42. Wang JY, Lee CH, Cheng SL, Chang HT, Hsu YL, Wang HC, et al. Comparison of the clinical manifestations of severe acute respiratory syndrome and mycoplasma pneumoniae pneumonia. J Formos Med Assoc. 2004;103:894-9.

43. Principi N, Esposito S. Macrolide-resistant mycoplasma pneumoniae: its role in respiratory infection. J Antimicrob Chemother. 2013;68:506-11.

44. Tamura A, Matsubara K, Tanaka T, Nigami H, Yura K, Fukaya T. Methylprednisolone pulse therapy for refractory mycoplasma pneumoniae pneumonia in children. J Inf Secur. 2008;57:223-8.

45. Tagliabue C, Salvatore CM, Techasaensiri C, Mejias A, Torres JP, Katz K, et al. The impact of steroids given with macrolide therapy on experimental mycoplasma pneumoniae respiratory infection. J Infect Dis. 2008;198:1180-8.

46. Luo Z, Luo J, Liu E, Xu X, Liu Y, Zeng F, et al. Effects of prednisolone on refractory mycoplasma pneumoniae pneumonia in children. Pediatr Pulmonol. 2014;49:377-80

\section{Publisher's Note}

Springer Nature remains neutral with regard to jurisdictional claims in published maps and institutional affiliations.

Ready to submit your research? Choose BMC and benefit from:
- fast, convenient online submission
- thorough peer review by experienced researchers in your field
- rapid publication on acceptance
- support for research data, including large and complex data types
- gold Open Access which fosters wider collaboration and increased citations
- maximum visibility for your research: over 100M website views per year
At BMC, research is always in progress.
Learn more biomedcentral.com/submissions

